\title{
Anti-N-Methyl-D-Aspartate Receptor Encephalitis Associated with Ovarian Teratoma in South China-Clinical Features, Treatment, Immunopathology, and Surgical Outcomes of 21 Cases
}

\author{
Huiyun Jiang $\mathbb{D}^{1},{ }^{1}$ Huixia Ye, ${ }^{1}$ Yifeng Wang, ${ }^{2}$ Yunhui Li, ${ }^{1}$ Ying Wang, $^{2}$ and Xiaomao Li $\mathbb{D}^{1}$ \\ ${ }^{1}$ Department of Gynecology, Third Affiliated Hospital, Sun Yat-sen University, Guangzhou 510630, China \\ ${ }^{2}$ Department of Gynecology and Obstetrics, Zhujiang Hospital, Southern Medical University, Guangzhou 510000, China \\ Correspondence should be addressed to Xiaomao Li; lixmao@mail.sysu.edu.cn
}

Received 30 March 2021; Accepted 8 May 2021; Published 21 May 2021

Academic Editor: Zhongjie Shi

Copyright (C) 2021 Huiyun Jiang et al. This is an open access article distributed under the Creative Commons Attribution License, which permits unrestricted use, distribution, and reproduction in any medium, provided the original work is properly cited.

\begin{abstract}
Objective. To study the clinical characteristics and surgical outcomes of anti-NMDAR encephalitis and the immunopathology of associated teratomas. Methods. Twenty-one patients were enrolled in this retrospective study, who were diagnosed with antiNMDAR encephalitis with ovarian teratoma and admitted to two tertiary hospitals in South China from July 2014 to December 2019. The clinical data of patients were reviewed. Comparisons were made between the patients with different outcomes after surgery. Immunohistochemical analyses of associated ovarian teratomas were performed. Results. The mean age of the patients was $24.33 \pm 5.12$ years. The peak seasons of disease onset were autumn and winter $(30.61 \%$ and $32.65 \%)$. The symptoms could be divided into 8 categories, including psychiatric abnormalities, seizures, movement dysfunction, consciousness disorders, autonomic dysregulation, speech disturbance, central hypoventilation, and memory deficits. All patients developed four or more categories of symptoms within the first four weeks. Twelve patients (57.1\%) had a maximum mRS of 5, and 11 patients (52.4\%) were admitted to ICU. Twenty patients received surgery, and only 3 patients were diagnosed pathologically with immature ovarian teratomas, while the other 17 patients had mature ovarian teratomas. After surgery, 17 patients (85.0\%) got clinical improvement. The central hypoventilation symptom and mature ovarian teratomas were associated with surgical outcome. Immunohistochemical analysis revealed that there were NMDAR-positive neural tissues in all 8 teratomas and in which 3 cases also contained large numbers of NMDAR-positive sebaceous glands and squamous epithelial tissues. Conclusion. The disease is of high prevalence in autumn and winter. The central hypoventilation symptom and mature ovarian teratomas were associated with surgical outcome. NMDAR-positive neural tissue is not the only etiological factor of encephalitis. We speculate that encephalitis development in some patients may result from NMDAR expression in sebaceous glands and squamous epithelial tissues.
\end{abstract}

\section{Introduction}

Anti-N-methyl-D-aspartate receptor (NMDAR) encephalitis belongs to immune-mediated disorder with prominent neuropsychiatric symptoms, which is serious and potentially lethal. It was firstly identified by Dalmau et al. [1]. Along with the progress of anti-NMDAR antibody detection and the growing attention paid to the disease, related researches began to rise rapidly. Several autoimmune diseases are known to have well-established seasonal variation. However, there is still little knowledge about the anti-NDMAR encephalitis's onset season.

Teratoma is a confirmed trigger of anti-NMDAR encephalitis [2]. About $50 \%$ of the female patients with antiNMDAR encephalitis were identified to have teratoma [3]. And Dalmau et al. [1] founded neural tissue with strong expression of NR2 subunits in the patients' teratoma, which could react with patients' antibodies. Thus, autoimmune factor is widely considered to be an important etiology of antiNMDAR encephalitis. However, some patients' teratomas 


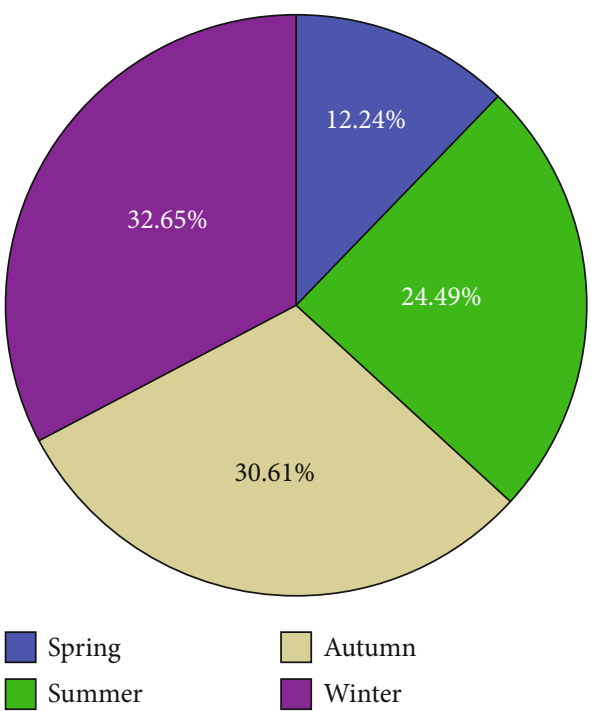

FIGURE 1: Seasonal variation of the incidence of anti-NMDAR encephalitis with ovarian teratoma.

lack neural tissue, and the immunopathology of the associated teratomas needs to be further studied.

The therapeutic methods of anti-NMDAR encephalitis involve immunotherapy and tumor section. First-line immunotherapy is the first choice, including corticosteroids, intravenous immunoglobulins, and plasma exchange. Second-line immunotherapy includes rituximab, cyclophosphamide, and mycophenolate mofetil [4]. Tumor section is usually recommended for patients with ovarian tumors, as studies reported that tumor section was associated with a lower rate of relapse in patients with teratoma compared to patients without teratoma [5]. But it is still lack of study focusing on the influence factors of surgical outcome.

This study is aimed at characterizing the onset season, clinical presentations, auxiliary examinations, treatment experience, immunopathology of teratomas, and prognosis of anti-NMDAR encephalitis with ovarian teratoma and comparing the difference in clinical features of patients with or without clinical improvement after surgery.

\section{Materials and Methods}

2.1. Patients. Patients diagnosed with anti-NDAMR encephalitis with ovarian teratoma, who were admitted into the Third Affiliated Hospital, Sun Yat-sen University, and Zhujiang Hospital, Southern Medical University, Guangzhou, China, from July 1, 2014 to December 30, 2019, were enrolled in this study, which was approved by the ethics committee of the Third Affiliated Hospital, Sun Yat-sen University (No. [2017]2-198). Written consent was provided by the patients or their representatives.

The inclusion criteria were as follows: (1) at least one of the symptoms, including psychiatric abnormalities, seizures, speech disturbance, dyskinesias, disturbance of consciousness, autonomic dysfunction, or central hypoventilation; (2) positive anti-NMDAR antibody in cerebrospinal fluid (CSF); (3) imaging findings of ovarian teratoma. All patients were diagnosed with anti-NMDAR encephalitis according to the criteria suggested by Dalmau et al. [2]. The fixed cellbased indirect immunofluorescence test (OUMENG FA $112 \mathrm{~d}-51$ ) was used to detect the anti-NMDAR antibodies in CSF or serum samples.

2.2. Data Collection. We collected demographic details, clinical features, auxiliary examinations and imaging examinations, treatments, and prognosis of patients included. Clinical features were divided into eight categories: psychiatric abnormalities, seizures, memory deficits, movement dysfunction, autonomic dysregulation, speech disorder, central hypoventilation, and loss of consciousness.

2.3. Outcome Evaluation. The modified Rankin Scale (mRS) was used to evaluate the severity of illness and outcome of patients $[4,6]$. An mRS score of $0-2$ was considered as good outcome.

A decrease in $\mathrm{mRS} \geq 1$ was defined as clinical improvement. No change in $\mathrm{mRS}$ or $\mathrm{mRS} \geq 4$ for 1 month after surgery was defined as having no clinical improvement. The new onset or worsening of symptoms occurring after two months of stabilization was considered as disease relapse [5]. Patients received prognosis evaluation at defined time points ( 1 week, 1 month, 3 months, 6 months, and 12 months after surgery).

2.4. Immunohistochemistry. Immunohistochemistry was performed on formalin-fixed, paraffin-embedded specimens. Slides of paraffin-embedded tumors were deparaffinized in xylene for $5 \mathrm{~min}$ and rehydrated in ethanol (4 min for $100 \%, 4 \mathrm{~min}$ for $95 \%, 4 \mathrm{~min}$ for $85 \%$, and $4 \mathrm{~min}$ for $70 \%$ ) and then washed by PBS for $2 \mathrm{~min}$. Sections were pretreated with EDTA buffer $\mathrm{pH} 8.0$ to retrieve antigens and washed three times with PBS. Sections were serially incubated with $3 \% \mathrm{H}_{2} \mathrm{O}_{2}$ for $10 \mathrm{~min}$ and blocked with goat serum (Boster, AR0009) for $30 \mathrm{~min}$. Sections were incubated at $4^{\circ} \mathrm{C}$ overnight with primary antibodies as follows: anti-NDMAR1 antibody ( $1: 200$; Abcam ab52177), anti-NMDAR2A ( $1: 25$; Abcam ab118587), anti-NMDAR2B ( $1: 25$; Novus NB10074475), and anti-MAP2(1:250; Abcam, ab183093). Then, sections were washed with PBS for three times and incubated with rabbit or mouse normal serum for $30 \mathrm{~min}$ and labeled polymer-HRP anti-rabbit or anti-mouse. DAB was used to stain the sections.

2.5. Statistical Analysis. SPSS version 20.0 was used to perform Statistical analyses. $p$ values $<0.05$ (two-sided) were considered significant. Baseline parameters were presented using descriptive statistics. Continuous variables were analyzed with an independent sample $t$-test. Contingency tables were analyzed using Pearson's chi-square test or Fisher's two-sided exact test when appropriate. Factors influencing surgical outcome were assessed by multivariable binary logistic regression.

\section{Results}

Twenty-one patients were reviewed, of which 15 patients were admitted to the Third Affiliated Hospital, Sun Yat-sen 
TABLE 1: General and clinical characteristics in patients having clinical improvement after surgery and patients having no improvement after surgery $(N=20)$.

\begin{tabular}{|c|c|c|c|c|}
\hline Parameters & $\begin{array}{l}\text { Patients having improvement after surgery } \\
\qquad(n=17)^{\S}\end{array}$ & $\begin{array}{c}\text { Patients having no improvement after } \\
\text { surgery }(n=3)^{€}\end{array}$ & $t$ & $p$ \\
\hline Age $(y)$ & $23.53 \pm 5.03$ & $28.00 \pm 5.56$ & -1.403 & 0.178 \\
\hline Fertilized women & $3(17.6 \%)$ & $2(66.7 \%)$ & & $0.140^{*}$ \\
\hline History of ovarian surgery & $2(11.8 \%)$ & $0(0.0 \%)$ & & $0.716^{*}$ \\
\hline \multicolumn{5}{|l|}{ Clinical features [5] } \\
\hline \multicolumn{5}{|l|}{ Prodromal symptoms } \\
\hline Fever $\left(T>37.5^{\circ} \mathrm{C}\right)$ & $4(23.5 \%)$ & $1(33.3 \%)$ & & $0.601^{*}$ \\
\hline Headache & $6(35.3 \%)$ & $2(66.7 \%)$ & & $0.620^{*}$ \\
\hline Initial symptoms & & & & $0.579^{*}$ \\
\hline Psychiatric abnormalities & $9(52.9 \%)$ & $2(66.7 \%)$ & & \\
\hline Seizures & $8(47.1 \%)$ & $1(33.3 \%)$ & & \\
\hline Psychiatric abnormalities & $17(100.0 \%)$ & $3(100.0 \%)$ & & - \\
\hline Seizures & $12(70.6 \%)$ & $2(66.7 \%)$ & & $0.681^{*}$ \\
\hline Movement dysfunction & $10(58.8 \%)$ & $2(66.7 \%)$ & & $0.656^{*}$ \\
\hline Loss of consciousness & $8(47.1 \%)$ & $3(100.0 \%)$ & & $0.218^{*}$ \\
\hline Autonomic dysregulation & $10(58.8 \%)$ & $1(33.3 \%)$ & & $0.566^{*}$ \\
\hline Speech disturbance & $6(35.3 \%)$ & $2(66.7 \%)$ & & $0.537^{*}$ \\
\hline Central hypoventilation & $5(29.4 \%)$ & $3(100.0 \%)$ & & $0.049^{*}$ \\
\hline Memory deficits & $2(11.8 \%)$ & $0(0.0 \%)$ & & $0.716^{*}$ \\
\hline Number of symptoms ${ }^{\#}[4]$ & $3.94 \pm 1.39$ & $5.33 \pm 1.15$ & -1.627 & 0.121 \\
\hline ICU admission [4] & $8(47.1 \%)$ & $3(100.0 \%)$ & & $0.218^{*}$ \\
\hline $\mathrm{mRS}$ at onset [4] & $4.23 \pm 0.90$ & $5.00 \pm 0.00$ & -3.490 & 0.003 \\
\hline $\begin{array}{l}\text { Diameter of the ovarian } \\
\text { teratoma }(\mathrm{mm})\end{array}$ & $48.47 \pm 35.31$ & $49.00 \pm 13.53$ & -0.025 & $0.980^{*}$ \\
\hline Pathological type & & & & $0.284^{*}$ \\
\hline Mature ovarian teratoma & $16(94.1 \%)$ & $2(66.7 \%)$ & & \\
\hline Immature ovarian teratoma & $1(5.9 \%)$ & $1(33.3 \%)$ & & \\
\hline Time until surgery initiation $(\mathrm{d})$ & $36.10 \pm 13.22$ & $45.67 \pm 21.22$ & -0.969 & $0.353^{*}$ \\
\hline
\end{tabular}

${ }^{\varsigma} \mathrm{A}$ decrease in $\mathrm{mRS} \geq 1$ was defined as having clinical improvement after surgery. ${ }^{\epsilon}$ No change in $\mathrm{mRS}$ or mRS $\geq 4$ for 1 month after surgery was defined as having no clinical improvement. ${ }^{*}$ The eight symptom categories are as follows: psychiatric abnormalities, seizures, movement dysfunction, loss of consciousness, autonomic dysregulation, speech disturbance, central hypoventilation, and memory deficits. *Fisher's exact test. mRS: modified Rankin Scale; ICU: intensive care unit.

University, and the other 6 patients were admitted to the Zhujiang Hospital, Southern Medical University, Guangzhou, China. All 21 patients were female. The mean age of patients was $24.33 \pm 5.12$ years (ranging from 15 to 33 years old). The disease could occur all year round, and the peak seasons focused in autumn and winter $(30.61 \%$ and $32.65 \%$ ) (Figure 1).

3.1. Clinical Features. Thirteen patients (61.9\%) had prodromal symptoms, including fever and headache, and the mean duration was $7.15 \pm 5.92 \mathrm{~d}$, ranging from $1 \mathrm{~d}$ to $20 \mathrm{~d}$. And 20 patients (95.2\%) had psychiatric abnormalities, 14 (66.7\%) patients had seizures, 12 (57.1\%) patients had movement dysfunction, $11(52.4 \%)$ patients had loss of consciousness, 11 (52.4\%) patients had autonomic dysregulation, 9 (42.9\%) patients had speech disturbance, $8(38.1 \%)$ patients had central hypoventilation, and 2 (9.5\%) patients had memory deficits. For all patients, 12 of them (57.1\%) developed psychiatric symptoms as initial manifestations, and the other 9 cases (42.9\%) presented with seizures initially. All patients developed four or more categories of symptoms within the first four weeks. Twelve of 21 patients $(57.1 \%)$ had a maximum mRS of 5, and 11 of 21 patients $(52.4 \%)$ were admitted to ICU.

Only one patient $(4.8 \%)$ had lower abdominal pain during the onset period, as a result of acute torsion of ovarian teratoma. Other patients did not present any gynecological symptoms.

3.2. Ancillary Examinations. Ten of 21 patients (47.6\%) had lymphocytic pleocytosis of cerebrospinal fluid. Seropositive anti-thyroid autoimmune antibodies were detected in 10 of 21 patients $(47.6 \%)$. Seven of 21 patients $(33.3 \%)$ had 
TABLE 2: Factors associated with clinical improvement after surgery (multivariable analysis).

\begin{tabular}{lccc}
\hline Factor & $p$ & OR & $95 \%$ CI \\
\hline Age & 0.160 & & \\
Fertilized women & 0.718 & & \\
Loss of consciousness & 0.089 & & \\
Central hypoventilation & 0.021 & 0.89 & $0.60-1.45$ \\
Number of symptoms & 0.109 & & \\
ICU admission & 0.089 & & \\
mRS at onset & 0.152 & & \\
Pathological type & & & \\
Mature ovarian teratoma & 0.007 & 1.39 & $0.89-1.93$ \\
\hline
\end{tabular}

OR: odds ratio; CI: confidence interval; mRS: modified Rankin Scale; ICU: intensive care unit.

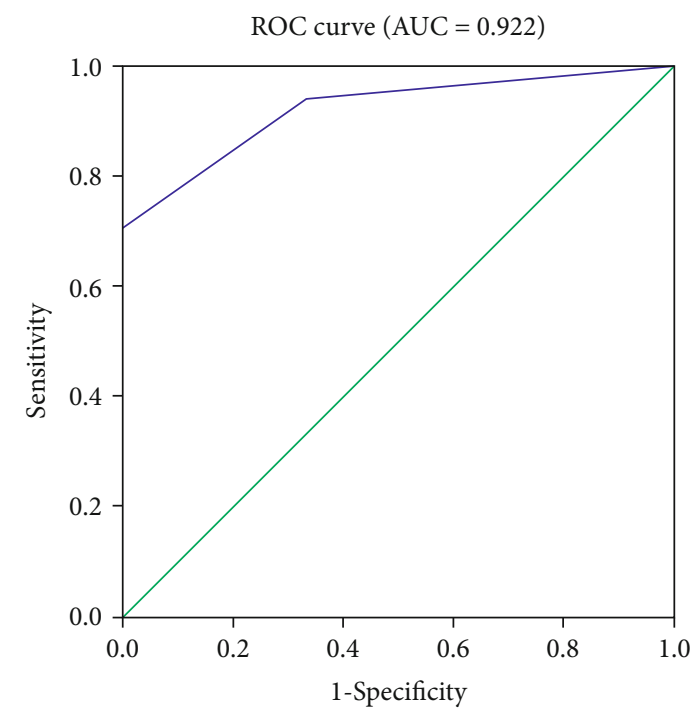

FIGURE 2: ROC curves showed the predictive efficiency of the model for the surgical outcome.

abnormalities in thyroid function test. In addition, 8 patients underwent 25-hydroxyvitamin $\mathrm{D}$ detection, and 7 of them (87.5\%) had a lower level than normal, ranging from 18.9 to $44.0 \mathrm{nmol} / \mathrm{L}$.

We detected serum cancer antigen 125 (CA 125), carbohydrate antigen 19-9 (CA 19-9), and serum alpha protein (AFP). And 8 of 21 patients' (38.1\%) CA 125 level was higher than normal, and the maximum value was $218.6 \mathrm{KU} / \mathrm{L}$. Three of the 21 patients (14.3\%) had elevated AFP levels, and the maximum value was $34.9 \mathrm{ng} / \mathrm{ml}$. All patients' CA19-9 values were in the normal range.

All patients received intensive oncological assessments routinely, including pelvic ultrasound and/or abdominalpelvis CT/MRI. The diameters of the ovarian teratoma ranged from 10 to $160 \mathrm{~mm}$. Nineteen of the 21 patients' (90.5\%) ovarian teratomas were unilateral, and only 2 of the 21 patients' $(9.5 \%)$ ovarian teratomas were bilateral.

3.3. Treatment and Outcomes. All patients received immunotherapy. Eight patients (38.1\%) were treated only with single or multiple first-line immunotherapies, and the other 13 patients $(61.9 \%)$ received first-line immunotherapies and second-line immunotherapy subsequently.

Twenty patients underwent surgery during the initial episode. Only one patient did not receive surgery because of the rejection of her family. Of the 20 patients with surgical treatment, 6 patients $(30.0 \%)$ received ovarian cystectomy, and the other 14 patients $(70.0 \%)$ received affected unilateral adnexectomy. The mean mRS score of patients receiving ovarian cystectomy was $3.50 \pm 0.84$, which was lower than that of patients receiving affected unilateral adnexectomy $(4.71 \pm 0.61)$ $(p=0.006)$. The mean duration between disease onset and surgery was $36.92 \pm 14.72 \mathrm{~d}$, ranging from $16 \mathrm{~d}$ to $63 \mathrm{~d}$. Of the 20 patients who underwent surgery, 17 patients $(85.0 \%)$ got clinical improvement after surgery, and the other 3 patients $(15.0 \%)$ had no improvement of the mRS scores for 4 weeks after surgery.

Table 1 summarized the difference of general and clinical characteristics of patients having clinical improvement and patients having no clinical improvement after surgery. There was no significant difference in age, gravidity, history of ovarian surgery, prodromal symptoms, type of initial symptoms, incidence of all other symptoms except central hypoventilation, number of symptoms, ICU admission, diameter of the ovarian teratoma, pathological type of ovarian teratoma, or duration between surgery and onset between patients having no clinical improvement after surgery and patients having clinical improvement after surgery. The incidence of central hypoventilation in patients having no clinical improvement after surgery was significantly higher than those in patients having clinical improvement after surgery (100.0\% vs. $29.4 \%$, $p=0.049)$. Additionally, the $\mathrm{mRS}$ score at acute onset in patients having no improvement after surgery was $5.00 \pm$ 0.00 , which was higher than that in patients having clinical improvement after surgery $(4.23 \pm 0.90)$, and the difference was statistically significant $(p=0.003)$. In multivariable analysis, the factors associated with surgical outcome included central hypoventilation (OR 0.89, 95\% CI 0.60-1.45, and $p=0.021$ ) and mature ovarian teratoma (OR 1.39, 95\% CI 0.89-1.93, and $p=0.007$ ) (Table 2). The estimated regression coefficients were as follows: $\operatorname{logit}(p)=-19.817^{*} A+2.079^{*} B+19.123$, where $A$ represented the symptom of central hypoventilation, and $B$ represented the pathological type of mature ovarian teratoma. The ROC curve revealed that the model was reliable in predicting the surgical outcome (Figure 2).

At 12-month follow-up, all of the 17 patients having clinical improvement after surgery had a good outcome $(\mathrm{mRS} \leq 2)$ and no one died or relapsed. Among 3 patients having no improvement after surgery, no one had a good outcome $(m R S \leq 2)$. No relapse of ovarian tumor or significant changes in menstrual pattern was observed in the 20 patients who underwent surgery.

3.4. Pathological Findings. Seventeen of the 20 patients' (85.0\%) histological types were reported to be mature ovarian teratoma, and the other $3(15.0 \%)$ were reported to be immature ovarian teratoma. We performed immunohistochemical tests for 8 of the 20 patients' teratoma tissues. The result revealed that all 8 samples were positive for MAP2, a specific 


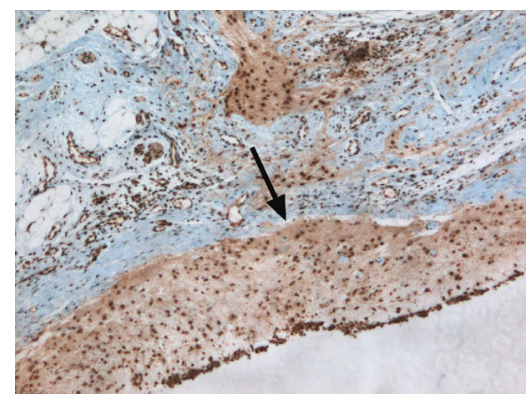

(a)

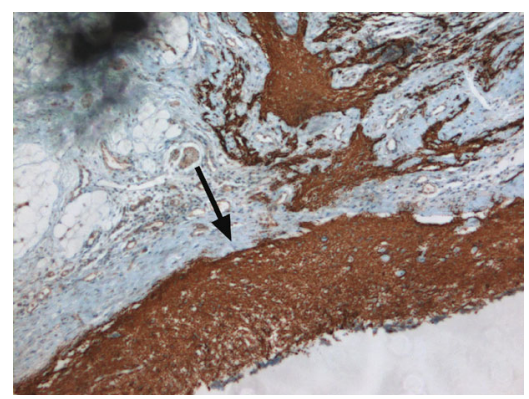

(c)

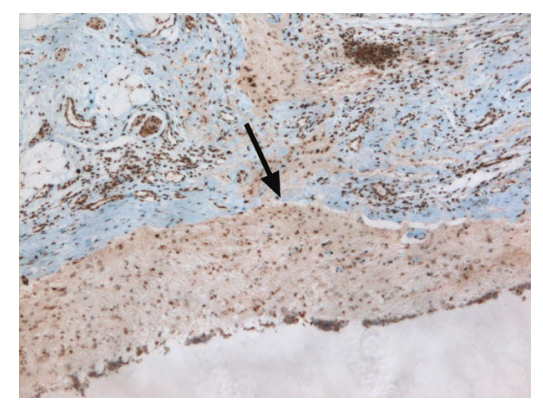

(b)

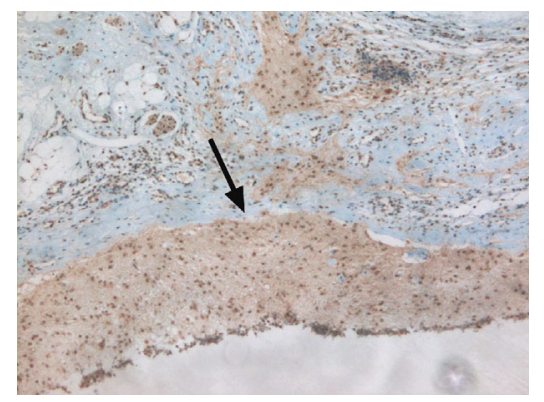

(d)

FIGURE 3: Representative immunohistochemical features. Densely aggregated neurons (the black arrow) were positive for (a) MAP2, (b) NDMAR1, (c) NDMAR2A, and (d) NDMAR2B (a-d, $\times 40)$.

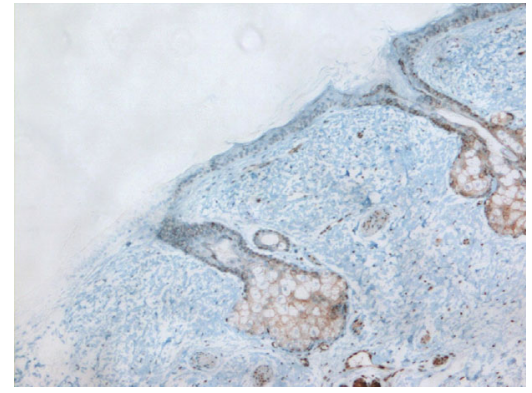

(a)

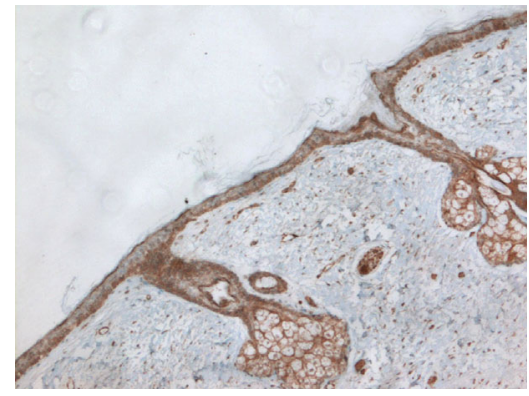

(c)

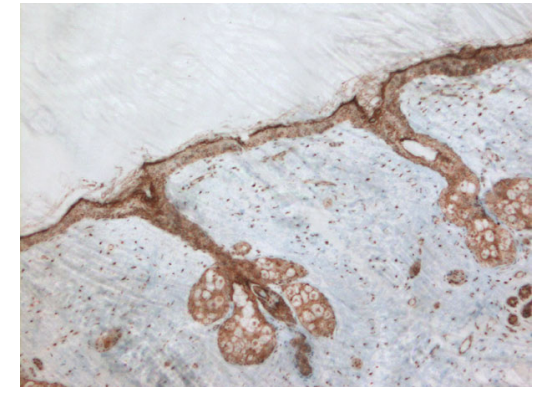

(b)

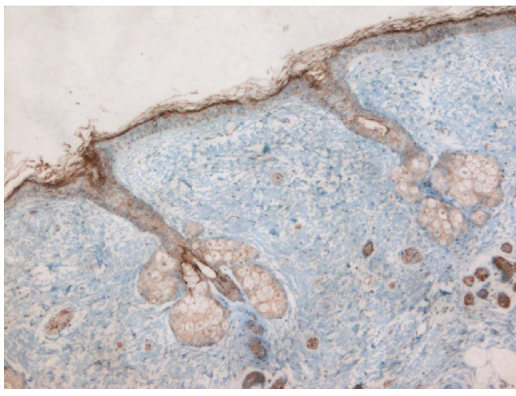

(d)

FIGURE 4: Representative immunohistochemical features. Sebaceous glands and squamous epithelial tissue were found in the tissue and were negative for (a) MAP2, strongly positive for (b) NMDAR1 and (c) NDMAR2A, and weakly positive for (d) NMDAR2B (a-d, $\times 40$ ).

marker of mature neurons, and the same areas were also positive for NDMAR1, NMDAR2A, and NMDAR2B (Figure 3). Among which, 3 patients' ovarian teratomas also showed positive for NDMAR1, NMDAR2A, and NMDAR2B in the sebaceous glands and squamous tissues (Figure 4).

\section{Discussion}

This retrospective study demonstrates that patients with anti-NMDAR encephalitis with ovarian teratoma tend to present more neuropsychiatric symptoms than gynecological 
symptoms. The disease incidence is higher during autumn and winter. Most patients get clinical improvement after surgery. The central hypoventilation symptom and mature ovarian teratomas are associated with surgical outcome. Partial cases also contain large number of NMDAR-positive sebaceous glands and squamous tissues.

The mean age of the patients in this study was consistent with other studies [5]. The youngest patient in our study was 15 years old. Leel et al. reported the youngest patient with anti-NDMAR encephalitis with ovarian teratoma, who was just 7 years old [7]. So it suggests that there is a need to conduct ovarian tumors screening for female patients of all ages.

Several autoimmune diseases are known to have wellestablished seasonal variation. The incidence of acute gouty attacks is highest in the spring. The onset or exacerbation of rheumatoid arthritis and Wegener's granulomatosis are all seen more commonly in the winter [8]. However, there were few studies focused on the onset season of antiNDMAR encephalitis. Adang et al. [9] revealed that antiNDMAR encephalitis without tumor were more common in warm months (April-September) (18/23, 78\%), whereas anti-NDMAR encephalitis with tumors presented during cold months $(6 / 6,100 \%)$. Our study revealed that the onset of anti-NMDAR encephalitis with ovarian teratoma was more commonly in autumn and winter, which was the same with the current study. As the samples of both studies are limited, we still need a larger-scale study to determine whether anti-NMDAR encephalitis has seasonal variation or not.

Our data revealed that most patients presented psychiatric abnormalities as initial symptom, which was consistent with previous studies [10-12]. As to the gynecological symptoms, only one patient had lower abdominal pain. It revealed that it is important to screen tumors with imaging methods for all anti-NMDAR encephalitis patients. Ultrasound is the first choice to screen ovarian teratoma, and transvaginal ultrasound is more sensitive than transabdomial ultrasound [13]. CT or MRI has a higher sensitivity in detecting teratomas, compared to that of ultrasound [14]. However, ultrasound has many advantages, like the lower cost, shorter examination time, and convenience for the patients with serious conditions. In some cases of anti-NMDAR encephalitis, whose screening revealed no specific clue for ovarian teratoma, an occult teratoma was found by histological examination after laparoscopy examination and ovariectomy [15]. However, we could only conduct laparoscopic exploration for patients with detection of ovarian teratoma by imaging examination, because of ethical considerations.

The seropositive rate of anti-thyroid autoimmune antibodies in our study was $47.6 \%$, which was higher than that of limbic encephalitis patients (33\%) and the general population(10-15\%) [16, 17]. Study found that anti-NMDAR encephalitis patients with seropositive anti-thyroid antibodies had a higher mRS score [18], but the relevant mechanism remains to be studied.

The role of B cells in the autoimmunity of anti-NMDAR encephalitis has become increasingly prominent [19-21]. Recently, the effect of vitamin D on immune cells has gradu- ally gained attention $[22,23]$. Vitamin D suppresses the proliferation and differentiation of $\mathrm{B}$ cells, resulting in a reduction of immunoglobulin secretion [24]. Our data found that the levels of 25-hydroxyvitamin D of most patients with anti-NMDAR encephalitis with teratoma were significantly lower than normal. It suggests that 25-hydroxyvitamin D may be an index to reflect the disease severity, which needs further study to confirm.

Early treatment and a lack of ICU admission are reported predictors of good outcome [4]. In our studies, the proportion of ICU admission was higher for patients having clinical improvement after surgery than that of the patients having improvement after surgery, but the difference had no statistical significance. Our findings suggested that the central hypoventilation symptom and mature ovarian teratomas were associated with surgical outcome of patients with anti-NMDAR encephalitis and ovarian teratoma.

Teratoma may contain tissues from all three germ layers, including ectoderm, mesoderm, and endoderm, and it can be either mature or immature. Bost et al. [25] reported that $11.8 \%$ of ovarian teratoma was immature in patients with anti-NMDAR encephalitis, which was associated with a higher risk of death. Patients with immature ovarian teratoma accounted for $10.0 \%$ in our study, but they did not present a higher mRS score or worse prognosis. In our study, all teratomas contained neural tissue, and immunohistochemical analysis confirmed that NMDAR expressed in the neural tissues, besides that NMDAR also expressed in the sebaceous glands and squamous tissue. As some patients' teratomas lack neural tissue and the majority of the teratomas are composed of skin, we speculate that encephalitis development in some patients may result from NMDAR expression in sebaceous glands and squamous epithelial tissues.

There were a few limitations in this study. First, this was a retrospective study and bias was inevitable. Second, our data only focused on the pathological results of ovarian teratoma in patients with anti-NMDAR encephalitis. Next, we plan to explore and compare the pathological features of teratomas in patients without anti-NDMAR encephalitis.

\section{Conclusion}

In summary, this study confirms that the disease incidence is higher during autumn and winter. Most patients get clinical improvement after surgery. The central hypoventilation symptom and mature ovarian teratomas are associated with surgical outcome. Systemic complications, such as pulmonary infection and respiration failure, should not be looked as surgical contraindications. We hope that our study can attract gynecologists and anesthesiologists' attention to the importance and necessity of early resection of tumors.

\section{Data Availability}

The data used to support the findings of this study are included within the article. 


\section{Conflicts of Interest}

The authors declare that there is no conflict of interest regarding the publication of this paper.

\section{Acknowledgments}

This work was supported by Wu Jieping Fund Medical Association (No. 320.6755.15012).

\section{References}

[1] J. Dalmau, E. Tüzün, H. Y. Wu et al., "Paraneoplastic anti-Nmethyl-D-aspartate receptor encephalitis associated with ovarian teratoma," Annals of Neurology, vol. 61, no. 1, pp. 25-36, 2007.

[2] J. Dalmau, T. Armangué, J. Planagumà et al., “An update on anti-NMDA receptor encephalitis for neurologists and psychiatrists: mechanisms and models," Lancet Neurology, vol. 18, no. 11, pp. 1045-1057, 2019.

[3] J. Dalmau, E. Lancaster, E. Martinez-Hernandez, M. R. Rosenfeld, and R. Balice-Gordon, "Clinical experience and laboratory investigations in patients with anti-NMDAR encephalitis," Lancet Neurology, vol. 10, no. 1, pp. 63-74, 2011.

[4] M. J. Titulaer, L. McCracken, I. Gabilondo et al., "Treatment and prognostic factors for long-term outcome in patients with anti- NMDA receptor encephalitis: an observational cohort study," Lancet Neurology, vol. 12, no. 2, pp. 157-165, 2013.

[5] Y. Dai, J. Zhang, H. Ren et al., "Surgical outcomes in patients with anti-N-methyl D-aspartate receptor encephalitis with ovarian teratoma," American Journal of Obstetrics and Gynecology, vol. 221, no. 5, pp. 485.e1-485.e10, 2019.

[6] N. Gresa-Arribas, M. J. Titulaer, A. Torrents et al., “Antibody titres at diagnosis and during follow-up of anti-NMDA receptor encephalitis: a retrospective study," Lancet Neurology, vol. 13, no. 2, pp. 167-177, 2014.

[7] N. Leel, H. S. Thakkar, D. Drake, and N. Bouhadiba, "Ovarian teratoma associated with anti-NMDA (N-methyl D-aspartate) receptor encephalitis," BMJ Case Reports, vol. 2018, p. 3, 2018.

[8] N. Schlesinger and M. Schlesinger, "Seasonal variation of rheumatic diseases," Discovery Medicine, vol. 5, no. 25, pp. 64-69, 2005.

[9] L. A. Adang, D. R. Lynch, and J. A. Panzer, "Pediatric antiNMDA receptor encephalitis is seasonal," Annals of Clinical Translational Neurology, vol. 1, no. 11, pp. 921-925, 2014.

[10] X. Liu, B. Yan, R. Wang et al., "Seizure outcomes in patients with anti-NMDAR encephalitis: a follow-up study," Epilepsia, vol. 58, no. 12, pp. 2104-2111, 2017.

[11] S. Sartori, M. Nosadini, E. Cesaroni et al., "Paediatric anti-Nmethyl-D-aspartate receptor encephalitis: the first Italian multicenter case series," European Journal of Paediatric Neurology, vol. 19, no. 4, pp. 453-463, 2015.

[12] M. J. Titulaer and J. Dalmau, "Seizures as first symptom of anti-NMDA receptor encephalitis are more common in men," Neurology, vol. 82, no. 7, pp. 550-551, 2014.

[13] S. Campbell and A. Gentry-Maharaj, "The role of transvaginal ultrasound in screening for ovarian cancer," Climacteric, vol. 21, no. 3, pp. 221-226, 2018.

[14] M. Saleh, P. Bhosale, C. O. Menias et al., "Ovarian teratomas: clinical features, imaging findings and management," Abdominal Radiology, 2021.
[15] A.-L. Boeck, F. Logemann, T. Krauß et al., "Ovarectomy despite negative imaging in anti-NMDA receptor encephalitis: effective even late," Case Rep Neurol Med, vol. 2013, article 843192, 3 pages, 2013.

[16] H. Pruss, J. Dalmau, L. Harms et al., "Retrospective analysis of NMDA receptor antibodies in encephalitis of unknown origin," Neurology, vol. 75, no. 19, pp. 1735-1739, 2010.

[17] C.-L. Xu, L. Liu, W.-Q. Zhao et al., “Anti-N-methyl-D-aspartate receptor encephalitis with serum anti-thyroid antibodies and IgM antibodies against Epstein-Barr virus viral capsid antigen: a case report and one year follow-up," BMC Neurology, vol. 11, 2011.

[18] Y. Lin, S. Tan, Y. Wang et al., "Anti-thyroid antibodies and thyroid function in anti-N-methyl-d-aspartate receptor encephalitis," Neurochemistry International, vol. 113, pp. 107-111, 2018.

[19] J.-P. Camdessanché, N. Streichenberger, G. Cavillon et al., "Brain immunohistopathological study in a patient with antiNMDAR encephalitis," European Journal of Neurology, vol. 18, no. 6, pp. 929-931, 2011.

[20] Y. Hachiya, A. Uruha, E. Kasai-Yoshida et al., "Rituximab ameliorates anti-N-methyl-D-aspartate receptor encephalitis by removal of short-lived plasmablasts," Journal of Neuroimmunology, vol. 265, no. 1-2, pp. 128-130, 2013.

[21] E. Martinez-Hernandez, J. Horvath, Y. Shiloh-Malawsky, N. Sangha, M. Martinez-Lage, and J. Dalmau, "Analysis of complement and plasma cells in the brain of patients with anti-NMDAR encephalitis," Neurology, vol. 77, no. 6, pp. 589-593, 2011.

[22] A. Caraba, V. Crişan, I. Romoşan, I. Mozoş, and M. Murariu, "Vitamin D status, disease activity, and endothelial dysfunction in early rheumatoid arthritis patients," Disease Markers, vol. 2017, Article ID 5241012, 7 pages, 2017.

[23] M. G. Petruzzelli, L. Marzulli, F. Margari et al., "Vitamin D deficiency in autism spectrum disorder: a cross-sectional study," Disease Markers, vol. 2020, Article ID 9292560, 5 pages, 2020.

[24] A. Boonstra, F. J. Barrat, C. Crain, V. L. Heath, H. F. J. Savelkoul, and A. O'Garra, "1alpha,25-dihydroxyvitamin d3 has a direct effect on naive $\mathrm{CD} 4^{+} \mathrm{T}$ cells to enhance the development of Th2 cells," Journal of Immunology, vol. 167, no. 9, pp. 49744980, 2001.

[25] C. Bost, E. Chanson, G. Picard et al., "Malignant tumors in autoimmune encephalitis with anti-NMDA receptor antibodies," Journal of Neurology, vol. 265, no. 10, pp. 2190-2200, 2018. 